\title{
EL FINANCIAMIENTO EDUCATIVO ARGENTINO EN LOS ÚLTIMOS TREINTA AÑOS (1989 - 2019) RUPTURAS Y CONTINUIDADES EN LAS TRES ÚLTIMAS DECADAS
}

\author{
Pedro Fiorucci * \\ Universidad Nacional de La Plata, Argentina \\ jpfiorucci@gmail.com \\ Alejandro Anton ** \\ Universidad Nacional de Rosario, Argentina \\ greenarrow87@gmail.com
}

Recibido: 17/06/2020 - Aceptado: 24/11/2020

\begin{abstract}
Resumen
El presente trabajo se propone realizar un análisis del financiamiento de la educación argentina atendiendo especialmente a la participación del Estado nacional en la inversión educativa, a través de las últimas tres décadas a fin de establecer contrapuntos sobre las rupturas y continuidades entre: el período neoliberal (1989 - 2001), el período de los gobiernos neodesarrollistas (2002 - 2015) y el período de gobierno iniciado con el ascenso de la Alianza Cambiemos al poder (2015 - 2019). Para realizar este análisis tendremos en cuenta el desempeño y las principales características del modelo de acumulación económica del país en cada periodo, y se utilizarán como indicadores estadísticos a la inversión educativa en porcentaje del PBI y la participación nacional y provincial dentro la inversión educativa. El uso de estos indicadores está en relación con la perspectiva de análisis que establece un vínculo entre la situación fiscal de los estados nacionales y provinciales, y su impacto en la inversión educativa. El abordaje metodológico es principalmente cuantitativo con el análisis de fuentes primarias de organismos del Estado, así como con fuentes secundarias de especialistas en financiamiento educativo.
\end{abstract}

Palabras clave: Financiamiento - Sistema educativo - Estado nacional - Provincias - Períodos políticos.

\section{ARGENTINE EDUCATIONAL FINANCING IN THE LAST THIRTY YEARS (1989 - 2019) BREAKS AND CONTINUITIES IN THREE POLITICAL CYCLES}

\begin{abstract}
This paper aims to carry out an analysis of the financing of Argentine education, paying special attention to the participation of the national State in educational investment, through the last three

\footnotetext{
*Profesor en Ciencias de la educación (UNLP). Doctorando en Ciencias de la Educación (Fahce-UNLP) con beca CONICET. Ayudante diplomado en la cátedra de Historia, Política y Gestión del Sistema Educativo (FaHCE - UNLP). Integrante de proyectos de investigación que abordan las configuraciones recientes de la carrera académica y la inserción laboral de los doctores de reciente formación en Argentina. A su vez es integrante de proyectos de extensión que abordan la articulación del nivel secundario y la educación superior.

** Licenciado en Ciencias de la Educación (FaHCE-UNLP). Doctorando en Ciencias Sociales (Fahce-UNLP) con beca CONICET. Ayudante diplomado en la cátedra de Epistemología de las Ciencias Sociales de la carrera de Licenciatura y Profesorado en Ciencias de la Educación (FaHCE-UNLP).
} 
REVISTA DE LA ESCUELA DE CIENCIAS DE LA EDUCACIÓN, AÑo 17, NRO. 16, VOL. 1, ENERO A JUNIO dE 2021. PÁGINAS 77-88. ISSN 2362-3349 (EN LíNEA). EL FINANCIAMIENTO EDUCATIVO ARGENTINO EN LOS ÚLTIMOS TREINTA AÑOS (1989-2019). RUPTURAS Y CONTINUIDADES EN LAS TRES ÚLTIMAS DECADAS. PEDRO, FIORUCCI. ALEJANDRO, ANTON.

decades in order to establish counterpoints on the ruptures and continuities between: the period of the neoliberal governments (1989 - 2001), the period of neo-development governments (2002 - 2015) and the period of government that began with the rise of the alliance Cambiemos to the power (2015 2019). To carry out this analysis, we will take into account the performance and main characteristics of the country's economic accumulation model in each period, and educational investment as a percentage of GDP, national and provincial participation in educational investment will be used as statistical indicators. The use of these indicators is related to the analysis perspective that establishes a link between the fiscal situation of the national and provincial states, and its impact on educational investment. The methodological approach is mainly quantitative with the analysis of primary sources from State agencies, as well as secondary sources from specialists in educational financing.

Keywords: Financing - Educational system - National state - Provinces - Political cycles.

\section{Introducción}

El financiamiento educativo en la Argentina se caracteriza por tener un esquema federal sobre una administración descentralizada. En este sentido, el Estado Nacional, las provincias y la Ciudad Autónoma de Buenos Aires son los responsables de la financiación del sector. Así es que existen tres fuentes para esta finalidad: los recursos del presupuesto nacional, los recursos provinciales producto de la recaudación tributaria y los recursos de la Coparticipación Federal de Impuestos. Este último resulta muy importante en la relación que se establece entre la nación y las provincias porque, por un lado, es un ingreso central para las provincias que vuelve muy dependientes a gran parte de ellas de estos recursos. Y, en segundo lugar, porque existe un desequilibrio con respecto a los recursos que se apropia la Nación en relación con las provincias siendo estas últimas quienes tienen más gastos respecto a sus recursos. A su vez este mecanismo no otorga la misma proporción a todas las provincias, sino que busca hacer una redistribución en favor de aquellas provincias más pobres, generando así situaciones más ventajosas o desventajosas para el sostenimiento de la educación en cada provincia (Morduchowicz, 2019).

En este sentido entendemos al financiamiento educativo, tanto a la fuente como a la forma de obtención de dinero, como un instrumento central de la política educativa que está regulado por el siguiente marco normativo: el Fondo de incentivo docente creado en 1999, y que fue prorrogado en 2004 por la ley №25.919, Ley de Garantía del Salario Docente № 25.864 del año 2003, Ley de Educación TécnicoProfesional N² 26.058 del año 2005, Ley de Financiamiento Educativo № 26.075 del año 2005 que también instituyó el Fondo de Compensación salarial docente y la Ley de Educación Nacional №26.206 del año 2006.

En este trabajo se van a comparar tres períodos con características particulares en la historia reciente argentina: el periodo neoliberal (1989 - 2001), el periodo neodesarrollista (2002-2015) y el periodo iniciado con el ascenso de Cambiemos al poder (2015 - 2019). Se intentará identificar tanto los puntos de continuidad como de ruptura entre los tres períodos como las modificaciones en cuanto al desempeño del modelo de acumulación económica que prima en cada periodo, al rol del Estado en las políticas públicas, y en particular en las políticas destinadas a educación. A modo de introducción a nuestra perspectiva de análisis, seguimos a Torrado (1992) cuando sostiene que el concepto modelo de acumulación, remite a "las estrategias de acción (objetivos, proyectos y prácticas políticas) relativas a los factores fundamentales que aseguran la acumulación capitalista y que son dominantes en una sociedad concreta en un momento histórico determinado" (s/p). A su vez, tomamos los aportes de Basualdo (2011) y Varesi (2011) que nos permiten entender el modelo de acumulación como la articulación de un determinado funcionamiento de las variables económicas, vinculado a una definida estructura económica, una peculiar forma de Estado y las luchas entre los bloques sociales existentes.

Nuestro análisis centra su atención en la participación del estado nacional en el financiamiento de la educación, lo cual no implica desconocer el importante peso que tienen las provincias en esta cuestión (sobre todo a partir de los cambios en los años noventa). Por otro lado, en este estudio analizamos la inversión a nivel macroeconómico y no nos detenemos en cómo se gastan esos recursos ni a qué sector (público o privado) afectan, aspecto que dejamos para posteriores indagaciones. Este trabajo es una primera sistematización del caso argentino, que busca indagar en esta cuestión desde un marco analítico que busca mirar el financiamiento educativo en relación con las orientaciones que asume el estado y el desempeño macroeconómico del país.

Los principales indicadores estadísticos que emplearemos para realizar este análisis serán la inversión educativa en porcentaje del PBI, participación nacional y provincial dentro la inversión educativa. El uso de estos indicadores está en relación con nuestra perspectiva de análisis, puesto que mostraremos el vínculo que hay entre la situación fiscal del Estado y su impacto en la inversión educativa, lo cual se vincula a nuestra hipótesis general de trabajo en la cual sostenemos que hay una relación particular entre el financiamiento del sector educativo y el desempeño y forma que asume el modelo de acumulación económica del país. Para hacer sustantivo el análisis con estos indicadores trabajaremos tanto con fuentes primarias de organismos del Estado, así como con fuentes secundarias de especialistas en financiamiento educativo.

El trabajo despliega tres grandes apartados en el que en cada período histórico seleccionado se analizan las principales tendencias del modelo de acumulación y las dinámicas del financiamiento educativo. A modo de cierre, en las conclusiones se presentan las líneas de continuidad y/o ruptura entre las tres etapas y algunas reflexiones sobre los desafíos futuros en el financiamiento del sector. 


\section{El período neoliberal. Reforma del Estado, cambio de paradigma educativo, protagonismo de las provincias en el financiamiento}

Cualquier intento por explicar el contexto social, político y económico de los años '90 no puede desvincularse de un proceso que comienza en 1976 con la última dictadura cívico militar. La razón de ello reside en que, es en este fatídico periodo donde se instauran un conjunto de políticas económicas y sociales profundamente regresivas que comienzan a transformar de modo radical nuestra sociedad. Principalmente destacamos la instauración, por medio de la violencia y la represión, de un patrón de acumulación económica nuevo: la valorización financiera, que colocó en el centro a la fracción financiera del capital y comenzó a desestructurar el modelo previo de industrialización por sustitución de importaciones (Basualdo, 2013). Estos elementos, articulados con el proceso de fuerte endeudamiento externo que comienza a acelerarse en este periodo y con la profundización de un patrón económico que tiende a concentrar el capital en sus beneficiarios en desmedro del trabajo, encendieron la mecha de un proceso que comenzaría a mostrar sus consecuencias en el mediano plazo.

$\mathrm{Si}$ bien el alfonsinismo intentó reeditar un esquema de cierta heterodoxia con políticas redistributivas sustentadas en un enfoque industrialista, cierto es que, por el carácter de la herencia de la dictadura (Forcinito y Tolon, 2009), así como por los diagnósticos con los que se decidió hacerle frente a este legado, el proyecto no prosperó. El corolario de este proceso fue una profunda recesión económica, un marcado crecimiento del desempleo y el trabajo informal, una crisis en el sector externo de la economía con declaración de cesación de pagos, una crisis hiperinflacionaria y la renuncia del presidente Alfonsín en 1989.

En este contexto asume Menem como presidente en el año 1989. El diagnóstico con el que decide hacer frente a los problemas sociales, políticos y económicos del país no tenía muchas distancias con las que se esgrimían en el último tramo del alfonsinismo. En este sentido, el diagnóstico sobre las causas de los problemas que acuciaban al país tenía como hipótesis un "estatismo ineficiente", "el despilfarro en el gasto", "el dirigismo" y el "facilismo" provocado por la intervención estatal (Forcinito y Tolón, 2009). Cabe ser destacado que este no fue un discurso puramente local, dado que sus principales voceros fueron los organismos multilaterales de crédito (Fondo Monetario Internacional y Banco Mundial), el gobierno de Estados Unidos y los medios de comunicación nacionales.

La síntesis de estas posturas es lo que se conoció como el consenso de Washington, que no era sino un proyecto que promovía una transformación de las relaciones entre política y economía en general, y en particular entre el Estado y el mercado. Las premisas básicas que sostenían el entramado de las propuestas del Consenso de Washington eran tres: 1) la reactivación económica de la región y su crecimiento sostenido dependen de los flujos de inversiones extranjeras; 2) para atraer esos flujos de inversiones los gobiernos deben brindar la mayor apertura posible a los mercados económicos, liberando para ello la economía de todo tipo de intervención estatal, puesto que ésta desvirtúa cualquier incentivo del mercado; 3 ) los gobiernos deben ejecutar amplias reformas estructurales de "libre mercado", eliminando controles, restricciones, subsidios y regulaciones. Estas directrices fueron la evidencia incuestionable que asumiría la racionalidad política del periodo (Vilas, 2007).

En consecuencia, el consenso reformista caló hondo e inició un nuevo proceso de reformas estructurales que continuó los cambios iniciados en la dictadura cívico militar. Las razones de por qué este consenso logró adhesión son múltiples, pero radican principalmente en las sucesivas crisis que fueron poniendo en jaque la estabilidad social, política y económica del país. Según Novaro (2010), los sucesivos picos inflacionarios ${ }^{1}$ que se dieron entre fines de los 80 s y principios de los ' 90 terminaron destruyendo la moneda como medio de intercambio y regulación económica; la recesión económica provocó un abrupto crecimiento de la pobreza, que para fines del año '89 se ubicaba en torno al $47 \%$; la deuda externa representaba para ese entonces el $70 \%$ del PBI, lo cual en la práctica implicaba la imperiosa necesidad de desfinanciar los gastos internos para saldar la deuda externa. En este contexto, la hipótesis de que el país resultaba ingobernable a causa del "estatismo intervencionista", y de que sólo volvería a ser gobernable a partir de aplicar estas reformas estructurales devino una certeza en gran parte de la clase política local.

En este contexto se sanciona la Ley de Reforma del Estado y la Ley de Emergencia Económica que oficiaron como el marco jurídico sobre el cual se realizaron una parte no menor de las transformaciones que se demandaban en el Consenso de Washington (Nahon, 2010). A estas medidas le siguió la Ley de Convertibilidad, que fue la respuesta que el gobierno ofreció ante el contexto hiperinflacionario de los años 89-91. La Ley de Convertibilidad implicó para el país la entrada en una nueva fase, la fase exclusivamente neoliberal del régimen de acumulación macroeconómica instaurado durante la dictadura cívico militar.

En lo efectivo, la convertibilidad implicó sostener un ancla cambiaria, es decir, sostener una paridad entre el peso nacional y el dólar norteamericano de $\$ 1$ a 1 USD. Esta medida se articuló con una apertura comercial prácticamente irrestricta. A su vez, el Estado Nacional delegaba históricas funciones en la determinación de los precios al control del mercado, con lo cual los precios internos fueron completamente disciplinados por el mercado. No obstante, la Convertibilidad como modelo socioeconómico manifestaba un esquema con evidentes tensiones y contradicciones internas que evidenciaban su poca sustentabilidad como modelo de integración social y reproducción económica. Este esquema de apertura económica a los flujos de capitales e importaciones internacionales era eminentemente deficitario en términos comerciales.

${ }^{1}$ Para el año 1989 se registró un índice anual del 3000\% de inflación. Para 1990 el registro se ubicó en 2300\%. 
Como no se podía financiar este esquema por medio del comercio externo se recurrió a dos estrategias distintas. En un primer momento, se financió por medio de la privatización de empresas y servicios públicos. Posteriormente, en un segundo momento, se financió en mayor medida a partir de un ciclo de endeudamiento externo, hasta que los pagos de los intereses de la deuda hicieron imposible sostener el esquema de compromisos asumidos, lo cual implicó una aceleración de las consecuencias negativas de este esquema macroeconómico que derivó en la crisis del año 2001.

Así, en términos generales, durante el periodo se privatizaron una cantidad no menor de activos del Estado Nacional, empresas públicas productoras tanto de bienes como de servicios públicos (salud, electricidad, gas, aerolíneas, etc.) y se desmontaron sendos mecanismos de regulación (mercado de finanzas, mercado de trabajo, etc.) con los que contaba el Estado Nacional para intervenir a nivel social, político y económico en la sociedad. No obstante, como sostienen los analistas del periodo, este conjunto de reformas no implicó por sí mismo la desaparición del Estado ni un achicamiento total. En todo caso, el achicamiento del Estado Nacional que se efectivizó fue en términos de su capacidad de regulación y sistema de gestión. Lo cual tuvo como contracara una concentración de poder político sin precedentes².

\section{Financiamiento del sistema educativo. Descentralización, disparidades en la coparticipación y aumento en la inversión educativa}

En sintonía con el proceso de reformas del Estado, el gobierno de Carlos Menem emprendió una profunda transformación educativa encaminada a colocar al sistema educativo sobre nuevas bases ${ }^{3}$. Varios autores ${ }^{4}$ señalan que los grandes impulsores intelectuales de estas reformas, a nivel internacional, fueron el Banco Mundial, el FMI y el Banco Interamericano de Desarrollo, que proponían el ajuste estructural del sistema como estrategias que contribuirían a paliar la crisis fiscal que atravesaba el Estado. Siguiendo estos postulados se tomaron medidas tendientes a descentralizar el sistema educativo a nivel provincial; se priorizó la educación primaria resignando recursos de la educación superior y se creó el sistema nacional de evaluación. En este sentido, los estados jurisdiccionales se hicieron cargo del financiamiento educativo mientras que el Estado nacional, por su parte, pasó a concentrar el poder de control y direccionamiento ${ }^{5}$.

A partir de la transferencia de las instituciones de nivel medio y superior no universitarias del Estado nacional a los estados provinciales y jurisdiccionales, se termina de federalizar el sistema educativo. Esta tendencia se había iniciado con las transferencias de las escuelas primarias desde nación a provincias en 1966 y que culminó en 1979. A partir de estos hechos, la suerte del financiamiento educativo pasó a estar centrada principalmente en la recaudación de impuestos coparticipables, ya que, si bien las provincias tienen otros medios de financiamiento, éstos tienen una participación mucho más reducida que los ingresos realizados por medio de la coparticipación.

El régimen federal de coparticipación genera una serie de disparidades (Rivas, 2010; Morduchowicz, 2002). Una forma específica de poder dimensionar estas desigualdades en el financiamiento educativo surge al comparar datos provinciales acerca de la inversión por alumno realizado por cada provincia y su densidad poblacional o entre el esfuerzo financiero que hace cada provincia para sostener la educación. De esto resulta que puedan existir provincias con características poblacionales semejantes, pero con dispersiones significativas en la inversión por alumno, así como que provincias con mayor participación en el PBI nacional tengan una inversión por alumno más reducido que otras con una participación menor, o que provincias con menores recursos tributarios hagan un esfuerzo financiero mayor en la inversión educativa.

Teniendo en cuenta estas disparidades en el financiamiento entre las jurisdicciones, nos centraremos ahora en el financiamiento educativo en relación con el PBI durante esta década, centrando la atención en la participación del Estado Nacional. Elegimos estos indicadores puesto que posibilitan ver la importancia que tuvo el sector en los diferentes periodos, así como la prioridad que cada nivel de gobierno le asignó.

\footnotetext{
${ }^{2}$ El marco legal en el que se sustenta la concentración de poder tiene como eje las leyes de Reforma del Estado, así como la Ley de Emergencia Económica.

${ }^{3}$ Dentro de las principales bases que orientaron la transformación del sistema destacamos: a) la Ley de Transferencia de Servicios Educativos del nivel medio y superior no universitario №24.049 de 1992; b) La Ley Federal de Educación N²4.195 de 1993, que plantea alcanzar el 6\% de inversión en educación como un objetivo; c) la Ley de Educación Superior №24.521 de 1995; d) El Pacto Federal Educativo de 1994, convertido en Ley №24.856 en 1997; y, por último, la reforma de la cláusula constitucional acerca de las leyes de organización y de base del sistema educativo sancionada por el Congreso.

${ }^{4}$ Suasnábar (1999); Feldfeber (2000); Saforcada (2012).

${ }^{5}$ Es esta una de las dimensiones que llevan a sostener a Tiramonti (2005) que la descentralización del sistema provocó una profundización del proceso de segmentación que atravesaba al sistema, derivando en una fragmentación del mismo.
} 
REVISTA DE LA ESCUELA DE CIENCIAS DE LA EDUCACIÓN, AÑo 17, NRO. 16, VOL. 1, ENERO A JUNIO DE 2021. PÁGINAS 77-88. ISSN 2362-3349 (EN LíNEA). EL FINANCIAMIENTO EDUCATIVO ARGENTINO EN LOS ÚLTIMOS TREINTA AÑOS (1989 - 2019). RUPTURAS Y CONTINUIDADES EN LAS TRES ÚLTIMAS DECADAS. PEDRO, FIORUCCI. ALEJANDRO, ANTON.

Cuadro 1. Composición de la inversión en Educación 1991-2002 como porcentaje del PBI y en millones de pesos constantes.

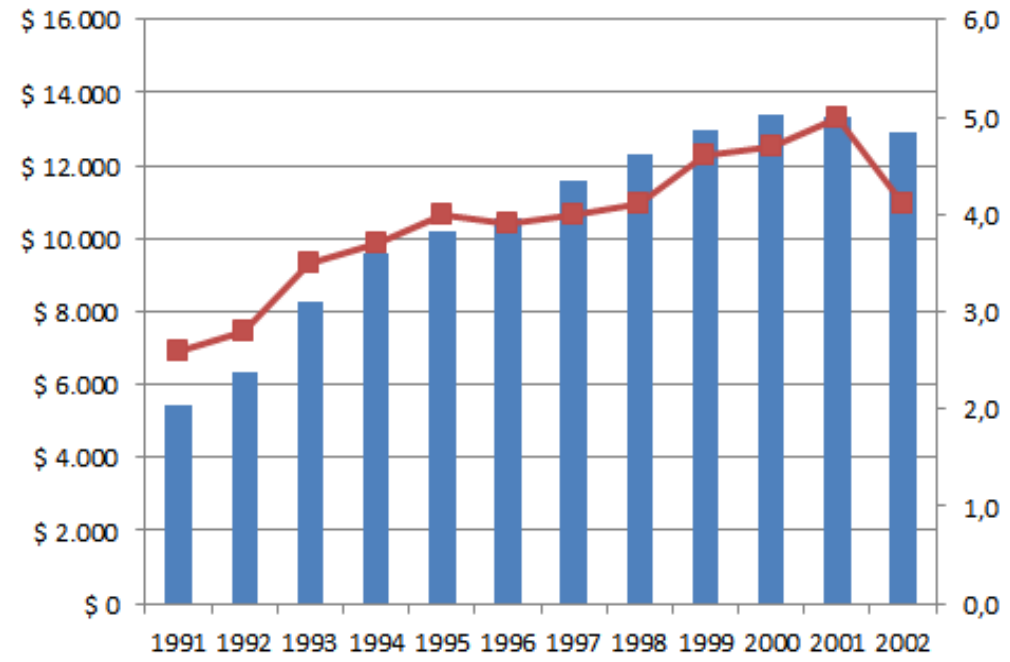

Fuente: Dirección de Análisis de Gasto Público y Programas Sociales - Secretaría de Política Económica

La trayectoria de la inversión educativa en la década, tal como muestra el cuadro 1, evidencia que parte de un porcentaje por debajo del recomendado ${ }^{6}$ por organismos internacionales, pero evidencia una constante suba, aún con todas las complejidades financieras que generó la transferencia de las instituciones del Estado Nacional a las provincias. Tanto en términos nominales, como en porcentaje del PBI, la inversión educativa muestra un movimiento ascendente que se interrumpe en el año 1996, continúa creciendo a partir de 1997 para registrar un pico de 5\% en el PBI en el año 2001, que luego cae en el 2002 a 4,1\% fruto del contexto económico local de crisis.

Cuadro 2. Participación porcentual de Jurisdicciones y el Poder Ejecutivo Nacional en la inversión consolidada en Educación.

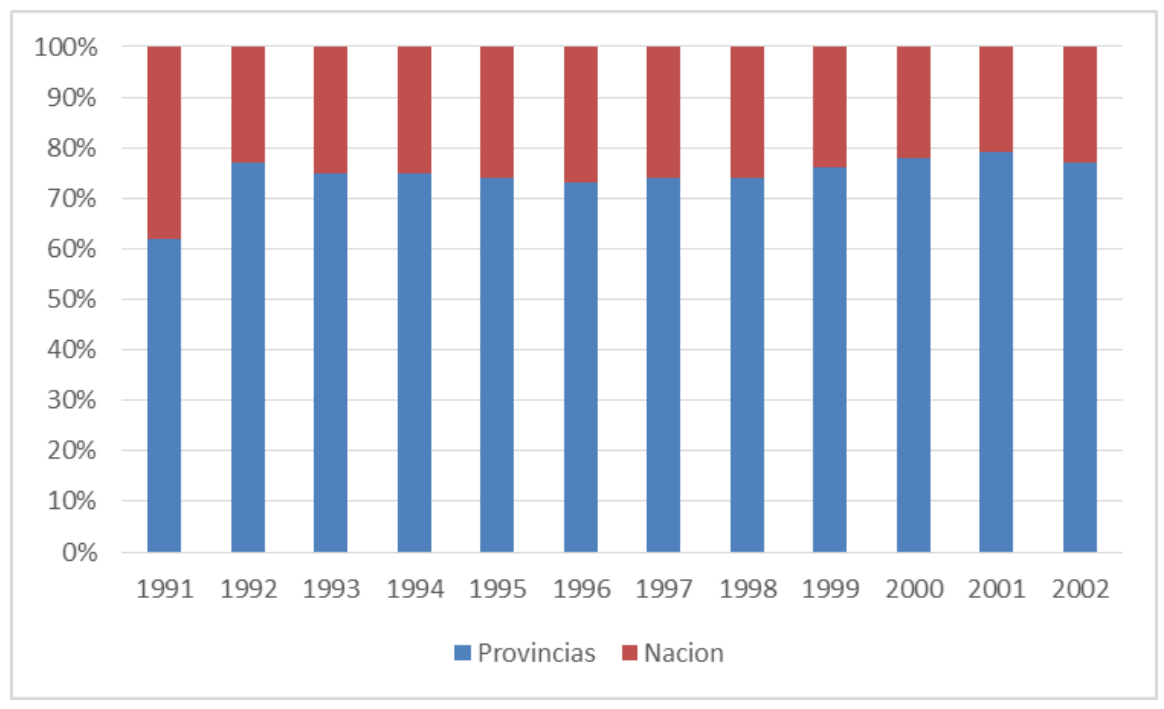

Fuente: Dirección de Análisis de Gasto Público y Programas Sociales - Secretaría de Política Económica.

El cuadro 2 muestra la participación que cada nivel de gobierno mantiene en la inversión educativa consolidado. En términos generales, el cuadro muestra que durante el periodo fue mucho más alta la participación en la inversión educativa de las jurisdicciones que el de la nación. De 1992 a 2002 mantienen una participación promedio del $75 \%$ en el financiamiento del sistema. El pico más alto de participación del

${ }^{6}$ UNESCO en el Marco de Acción Educación 2030 propuso dos patrones de referencia: destinar al menos de un $4 \%$ a un $6 \%$ del PIB a la educación, y/o destinar al menos de un $15 \%$ a un $20 \%$ de la inversión pública a la educación. 
REVISTA DE LA ESCUELA DE CIENCIAS DE LA EDUCACIÓN, AÑo 17, NRO. 16, VOL. 1, ENERO A JUNIO dE 2021. PÁGINAS 77-88. ISSN 2362-3349 (EN LíNEA). EL FINANCIAMIENTO EDUCATIVO ARGENTINO EN LOS ÚLTIMOS TREINTA AÑOS (1989-2019). RUPTURAS Y CONTINUIDADES EN LAS TRES ÚLTIMAS DECADAS. PEDRO, FIORUCCI. ALEJANDRO, ANTON.

gobierno nacional se registra en 1991, justo antes de realizarse la transferencia de las instituciones a las provincias. A partir de ahí, el gobierno nacional financiaría casi una cuarta parte del sistema educativo nacional, delegando el resto a las jurisdicciones.

\section{El período neodesarrollista. Reposicionamiento estatal, recomposición de capacidades dañadas y nuevas orientaciones en el financiamiento educativo}

A la luz del agotamiento del proyecto neoliberal que manifestó su crisis en el año 2001 con graves consecuencias políticas, económicas y sociales, se inicia un nuevo período político y económico en la Argentina. En el año 2002, a partir de un conjunto de políticas económicas empleadas por el presidente Eduardo Duhalde, se inicia el ciclo económico de la posconvertibilidad. La devaluación aplicada a la moneda nacional respecto al dólar, la implementación de retenciones a la exportación, el "salvataje" al capital financiero, el default económico, y el congelamiento y renegociación de las tarifas públicas fueron algunas de las principales políticas económicas que inauguraron el periodo (Varesi, 2011). Esto permitió darle una cierta estabilidad a la economía nacional, que heredaría el gobierno de Néstor Kirchner.

Así, con este conjunto de políticas comenzó a rearticularse una modalidad distinta en el modelo de acumulación económica del país tanto en su orientación exportadora como en la orientación que asumió hacia el mercado interno, que permiten definirlo, siguiendo a Varesi (2018) como un periodo de marcado tinte neodesarrollista. Este último se fue recomponiendo lentamente a través del mejoramiento paulatino de las capacidades de consumo de la población, así como de la capacidad industrial del país a partir de un conjunto de políticas que priorizaron al sector. Una de ellas, central en la recomposición industrial, fue la política cambiaria que se sostuvo en un tipo de cambio real alto, que ofició como medida de protección para este sector, así como un impulso a la fracción exportadora. La recomposición de ambos sectores posibilitó el mejoramiento de los indicadores de empleo, lo cual tuvo un efecto de retroalimentación en la dinámica general del modelo en curso.

Con estos pilares económicos, políticos y sociales, comenzó una etapa de crecimiento económico que entre el periodo 2003-2011 registró un crecimiento promedio de 7 puntos del PBI y que, incluso, contó con superávits gemelos (fiscal y comercial). A diferencia del periodo de la convertibilidad económica, donde el crecimiento económico tenía como grandes impulsores al sector financiero y de servicios, lo cual se evidenciaba en la baja capacidad para generar empleo en el periodo, en la posconvertibilidad el sector productivo tuvo un papel más protagónico (Basualdo, 2011)

No obstante, estas transformaciones, las continuidades con el periodo precedente fueron varias. Un rasgo de continuidad notable es la permanencia de una tendencia que atravesó el periodo neoliberal y que se consolidó en el período neodesarrollista, como la centralización y extranjerización de la economía local, la cual mantiene vigente una de las principales causas de grandes y recurrentes desequilibrios de la economía local; también destaca la permanencia de una estructura impositiva regresiva que grava en mayor medida los consumos y no las riquezas, así como la continuidad en manos privadas de servicios públicos que fueron privatizados en el periodo de la convertibilidad (Varesi, 2011).

Como sostiene Aronskind (2015), uno de los principales logros de las administraciones kirchneristas fue la capacidad que adquirió el Estado nacional de autofinanciamiento libre de las tutelas de los capitales privados extranjeros o locales. Esta capacidad adquirida por el Estado fue uno de los pilares que dotó de sustentabilidad económica a las políticas redistributivas empleadas en el periodo y que implicó una notoria diferencia con el modelo de la convertibilidad. Destacamos este aspecto, puesto que lo consideramos un elemento de suma importancia en una cuestión como el financiamiento educativo.

\section{Financiamiento del sistema educativo. Nuevo marco normativo y planificación de la inversión en el sector}

En el marco regional de un proceso de reformas que reposicionaron al Estado nacional como garante de derechos educativos y que elaboraron fuerte críticas hacia las políticas del período neoliberal, durante la presidencia de Néstor Kirchner (2003-2007) y las dos presidencias de Cristina Fernández de Kirchner (2007 - 2015) se sancionan varias leyes relativas al campo educativo que marcaron un cambio en el rumbo de las políticas ${ }^{7}$. A pesar de ello, no se modificó la estructura federal educativa que se consolidó con la descentralización de los años ' 90 . En este período se avanzó en la conceptualización de la educación como bien público y como derecho social a la par que se buscó consolidar la centralidad del Estado en la garantía de este derecho en los distintos niveles del sistema.

La Ley de Financiamiento Educativo 26.075 (LFE) es parte de este conjunto de leyes que intenta reorganizar el sistema educativo. En este sentido, su sanción como ley en el 2005, con vigencia a partir del año 2006, introdujo reglas de juego nuevas para coordinar la inversión que se destina a Educación, Ciencia y Tecnología entre los distintos niveles de gobierno con el fin expreso de llevar el financiamiento sectorial del 4,2\%, que registraba en el año de inicio, hacia el 6\% del PBI en el año 2010 (Art. 3), lo que no es otra

${ }^{7}$ Entre las leyes más relevantes destacamos: Ley de Garantía del salario docente y 180 días de clase ( $N^{\circ} 25.864$, año 2003), Ley de Fondo Nacional de Incentivo Docente ( $N^{\circ} 25.919$, año 2004), Ley de Educación Técnico Profesional $\left(N^{\circ}\right.$ 26.058, año 2005), Ley de Financiamiento Educativo ( $N^{\circ} 26075$, año 2005) que instituyó el Fondo de Compensación salarial docente, Ley Nacional de Educación Sexual Integral ( $N^{\circ} 26.150$, año 2006), Ley de Educación Nacional ( $\mathrm{N}^{\circ}$ 26.206, año 2006). Amparados en la ley 26075 y 26206 se convocó a la primera Paritaria Nacional Docente en el año 2008. Por último, se sancionó la ley de Responsabilidad del Estado en el nivel de educación superior (Ley 27.204, año 2015). 
cosa que elevar los recursos del sector en un 50\% aproximadamente. Es importante considerar que destinar el $6 \%$ del PBI al sector educativo no constituye, estrictamente una novedad en la política del sector. Este monto se encontraba estipulado en los términos establecidos por la Ley Federal de Educación de 1993, pero allí no estaban definidos las responsabilidades que cada nivel de gobierno tenía que asumir, ni la estrategia para llevarlo adelante. Por otro lado, como es posible apreciar en los cuadros estadísticos, la cifra nunca fue alcanzada.

Esta nueva legislación se orientó hacia la fijación de una estrategia, con múltiples dispositivos, que buscaron posibilitar la inversión educativa a la tan deseada meta del $6 \%$ del PBI. Asimismo, la ley avanzó en la fijación de los criterios que deberían cumplir en el financiamiento del sector tanto el gobierno central como los gobiernos jurisdiccionales, marcando otra notoria diferencia con la legislación anterior e iniciando un marco de planificación para la inversión en el sector.

El establecimiento de porcentajes financieros para cada nivel de gobierno está en función de materializar los objetivos educativos que contempla la ley en su artículo 2 . De este modo, el $40 \%$ de la pauta de aumento que deberá aportar el gobierno nacional tiene como finalidad atender principalmente a los objetivos contemplados para el sistema universitario y los del sistema científico-tecnológico nacional (Art. 4). Las provincias y la CABA con su $60 \%$ se orientarían a posibilitar la concreción de los objetivos referidos a mejorar la remuneración docente, adecuar la planta a la creciente matrícula, y jerarquizar la carrera docente (Art. 5). En cuanto a la fuente de los recursos económicos, la ley establece en su artículo 7 que ellos se obtendrán a partir de la afectación de la recaudación de impuestos coparticipables.

La ley no sólo establece dispositivos para incrementar de modo coordinado en los distintos niveles de gobierno y año a año el financiamiento sectorial, sino que fija también dispositivos para evitar que el financiamiento educativo no corra ningún riesgo de disminuir su participación en términos de porcentaje del producto como históricamente sucedió.

Sin embargo, la nueva normativa es puesta en cuestión desde distintos ángulos. En este sentido, varios autores han señalado aspectos frágiles en la Ley de Financiamiento 26.075. En primer lugar, Vera y Bilbao (2010) y Rivas (2010) señalan que, si bien la ley establece objetivos financieros y también educativos, respecto a los primeros los últimos tienen un elevado nivel de generalidad que dificulta su concreción. A su vez, señalan que difícilmente pueda solucionar la situación de injusticia estructural que genera el particular funcionamiento del federalismo argentino. Por otro lado, esta ley tenía un plazo de cumplimiento hasta el año 2010 y luego de ese año quedó regulado el financiamiento bajo la Ley de Educación Nacional (LEN), donde se estableció que la inversión del $6 \%$ debía ser únicamente para la cartera educativa sin considerar a Ciencia y Tecnología ${ }^{10}$, pero sin fijar una estrategia económica ni las responsabilidades que le corresponderían a cada nivel de gobierno. Esto implicó un retroceso en la planificación de la inversión hacia el sector, dejando el marco legal para esta área en un estado de cierta discrecionalidad similar a la legislación de los años '90 (Morduchowicz, 2019).

La actividad económica del país, tal como muestran Rivas y Bvorkin (2018), registró un crecimiento promedio cercano al 7\% año a año en términos del PBI durante el periodo de vigencia de la LFE (20062010). Sólo en el año 2009 hubo una caída, en relación con el promedio registrado, a partir de la crisis económica externa. Los demás años se mantuvieron dentro de un considerable crecimiento económico. El movimiento ascendente de este indicador no sólo significa mayor actividad económica, sino que en términos de la LFE implica un crecimiento efectivo de la inversión consolidada en educación, ciencia y tecnología, en tanto al crecer el producto interno el porcentaje de inversión que se le asigna al sector también crece dado que está ligado a su desempeño.

\footnotetext{
${ }^{10} \mathrm{Hacia} 2007$ se crea el Ministerio de Ciencia Tecnología e Innovación Productiva (MinCyT) y unos años más tarde, con
} el lanzamiento del Plan Innovadora 2020, se establece la meta de inversión del 1\% en relación al PBI para este sector. 
REVISTA DE LA ESCUELA DE CIENCIAS DE LA EDUCACIÓN, AÑo 17, NRO. 16, VOL. 1, ENERO A JUNIO dE 2021. PÁGINAS 77-88. ISSN 2362-3349 (EN LíNEA). EL FINANCIAMIENTO EDUCATIVO ARGENTINO EN LOS ÚLTIMOS TREINTA AÑOS (1989 - 2019). RUPTURAS Y CONTINUIDADES EN LAS TRES ÚLTIMAS DECADAS. PEDRO, FIORUCCI. ALEJANDRO, ANTON.

Cuadro 3. Inversión total consolidada en educación como porcentaje del PBI (2004 - 2015)

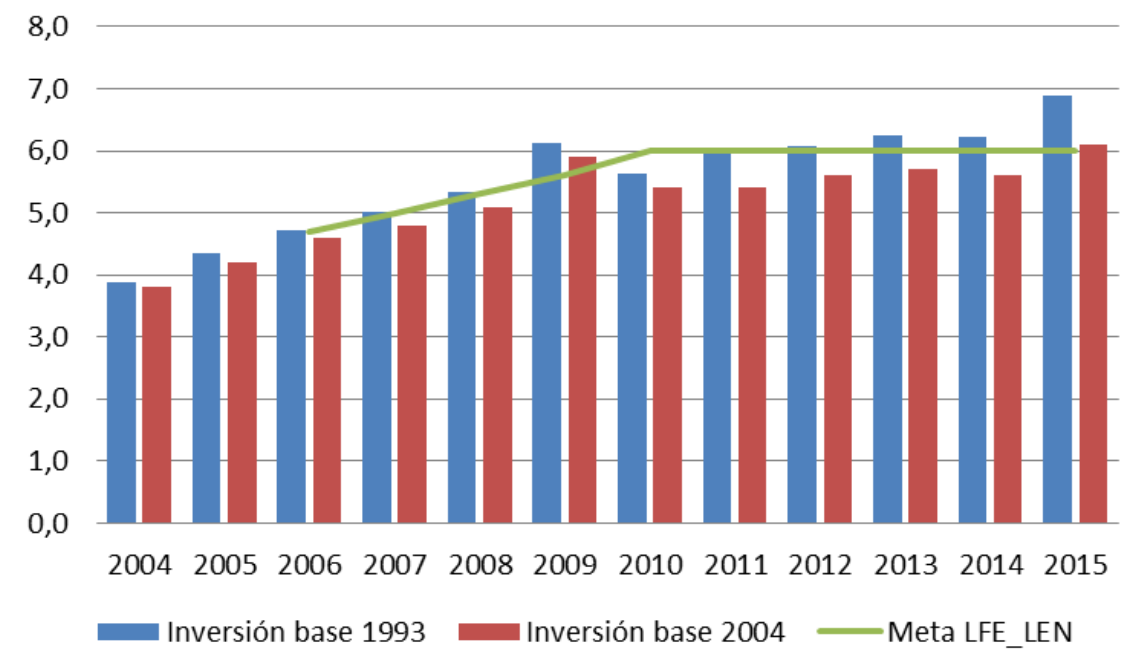

Fuente: Elaboración propia en base a los datos de la Secretaría de Política Económica, del Ministerio de Hacienda

A partir de mostrar las bases económicas sobre las que se sostuvo la LFE es posible analizar el desempeño de las que consideramos sus principales metas financieras. El cuadro 3 aporta datos para seguir la inversión educativa en términos de porcentajes del PBI. Así puede decirse que hasta 2008 se mantuvo la inversión en Educación, Ciencia y Tecnología en niveles superiores al 6\% del PIB base 1993 y algo inferior según el PIB base 2004. Hacia el año 2009 se cumple la meta considerando ambas bases y en el 2010 cae la inversión por debajo de la meta. A partir del 2011, con el cambio a la LEN que exigía el $6 \%$ de inversión sobre el PBI solo para educación (excluyendo a ciencia y tecnología), se cumple con el $6 \%$ según la base 1993, pero queda por debajo hasta el 2014. En 2015 vuelve a cumplirse la meta considerando ambas bases. Así puede sostenerse que hubo una tendencia de crecimiento, pero que solo se cumple la meta en ambas bases en 2009 y 2015.

Cuadro 4. Inversión consolidada en educación como porcentaje del PBI por nivel de gobierno

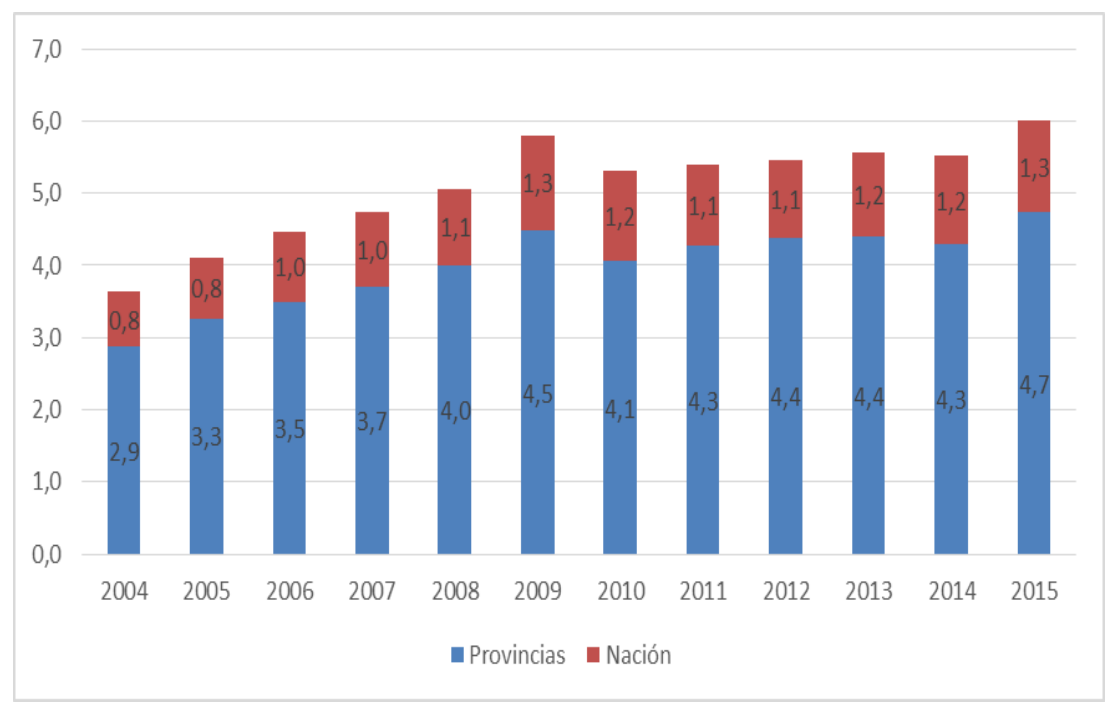

Fuente: Elaboración propia en base a los datos de la Secretaría de Política Económica, del Ministerio de Hacienda 
En la participación efectiva de cada nivel de gobierno en la inversión educativa, la pauta de inversión que debía incrementarse año a año, hasta alcanzar el 6\% del PBI en el año 2010, debía ser financiado en un $60 \%$ por las provincias y en un $40 \%$ por el gobierno nacional. Tal como se muestra en el cuadro 4, si bien el gobierno nacional sostuvo un $22 \%$ de financiamiento, y no el $40 \%$, se logró un incremento en el porcentaje de inversión en el sector, pero esto se debe a que el conjunto de provincias superó holgadamente el cumplimiento de sus metas de financiamiento. A partir de las desigualdades provinciales que expusimos, es posible identificar un conjunto de provincias que no logró cumplir las metas de la LFE, otras que las cumplieron con creces y otras que las cumplieron sin margen de sobrecumplimiento alguno (Bezem, 2012).

\section{El gobierno de la alianza Cambiemos. Nuevo bloque de poder en el Estado, promesas de revolución educativa y ajuste en el financiamiento del sector}

En diciembre de 2015 la alianza Cambiemos llega a la presidencia del poder ejecutivo. Desde entonces es posible registrar que tanto a nivel político y económico se vislumbra la conformación de un nuevo bloque de poder con un proyecto integral que se propone redefinir la estructura y funciones del Estado, así como modificar la distribución del ingreso de manera contundente (Basualdo y Manzanelli, 2016; Bona, 2016; CIFRA, 2016).

A nivel político son múltiples los cambios implicados por el arribo de la Alianza Cambiemos al gobierno. Entre ellos destacamos el hecho de que, por primera vez en la historia moderna del país, los sectores dominantes acceden al gobierno del Estado mediante un partido propio y el voto democrático. Esta novedad, lejos de representar un "dato de color" del nuevo gobierno que asume a finales de 2015, es un elemento que anticipa el cariz que comienza a exhibir el modelo de acumulación en Argentina, el tipo de Estado y las políticas públicas que éste implementará. En este sentido, la alianza Cambiemos implica un giro copernicano en el desarrollo social argentino, en tanto configura un nuevo bloque en el poder en el cual la hegemonía política la ostentan los bancos transnacionales y el capital extranjero en su conjunto. En cambio, los grandes sectores industriales y agrarios representantes del capital local integran el bloque, pero de modo subordinado (Cifra, 2016; Bona, 2016). Un breve repaso de las políticas realizadas al poco tiempo de asumir el poder ejecutivo la alianza Cambiemos posibilita vislumbrar los cambios en los elementos mencionados.

Como sostiene Varesi (2016), la política económica que dio inicio al programa económico del periodo fue la desregulación del mercado cambiario y una devaluación del 40\%, que en marzo de 2016 llegó al $60 \%$. A ello le siguió una baja en las retenciones a productos exportables, eliminación de distintos tipos de tributos a las exportaciones de productos agropecuarios, industriales y mineros. En el plano financiero se operó una fuerte suba en las tasas de interés, se posibilitó la liberalización en el movimiento de capitales, acompañado de la desregulación de distintos mercados (sector financiero, hidrocarburos, aseguradoras). Por el lado del comercio exterior, se operó una asimétrica apertura económica, que redundó en un cuantioso ingreso de importaciones. Todos estos cambios se aplicaron junto a fuertes aumentos en las tarifas de servicios públicos y en los combustibles. Por último, es menester reconocer que este conjunto de políticas orientadas a interpelar a los mercados fue acompañado por tenues medidas a favor de sectores sociales y asalariados como las transferencias, por única vez, a beneficiarios de la AUH, devolución del IVA a jubilados, y la ampliación de la AUH a monotributistas.

En los hechos, este conjunto de medidas implicó un fuerte shock ortodoxo de carácter neoliberal. Todo el conjunto de medidas posibilita ver un núcleo común: la redefinición del tipo o naturaleza de Estado. Se trata de lograr una modificación radical respecto a la estructura estatal heredada y reemplazar las políticas de corte regulatorio en diversos planos (cambiario, comercio exterior, precios, inversiones, etc.) por el libre juego de la oferta y la demanda, por la delegación de la regulación económica en las fracciones del capital que poseen una mayor incidencia en la estructura económica del país.

Estas medidas no son azarosas. La alianza Cambiemos llegó al gobierno con el diagnóstico de que los problemas macroeconómicos existentes al momento de asumir se debían, principalmente, a tres factores: 1) la excesiva intervención estatal, 2) la sobre expansión del consumo interno en base al nivel del salario de los trabajadores y 3) el elevado gasto público. Consecuente con este planteo, se propuso el objetivo explícito de poner en marcha un ajuste en el proceso económico. Y eso es lo que grafican el conjunto de políticas mencionadas.

En línea con este diagnóstico, la Alianza Cambiemos inició un nuevo ciclo de endeudamiento que se abrió con el pago de la deuda externa litigada por los "holdouts". Esta decisión vuelve a introducir a la economía dentro de un proceso de valorización financiera acompañado por un fuerte proceso de ajuste de la economía interna. Ya a principios de 2019 la ratio de endeudamiento, tanto con agentes privados externos como órganos de financiamiento internacional (como el FMI), comienza a presionar fuertemente los recursos con los que cuenta el Estado para cubrir sus gastos, entre los que se encuentra el financiamiento educativo. 
REVISTA DE LA ESCUELA DE CIENCIAS dE LA EdUCACIÓN, AÑo 17, NRO. 16, VOL. 1, ENERO A JUNIO DE 2021. PÁGINAS 77-88. ISSN 2362-3349 (EN LíNEA). EL FINANCIAMIENTO EDUCATIVO ARGENTINO EN LOS ÚLTIMOS TREINTA AÑOS (1989 - 2019). RUPTURAS Y CONTINUIDADES EN LAS TRES ÚLTIMAS DECADAS. PEDRO, FIORUCCI. ALEJANDRO, ANTON.

\section{Financiamiento del sistema educativo. Promesas de revolución educativa, ajuste por doble vía y endeudamiento}

Durante la campaña presidencial de Mauricio Macri se anunciaba un claro interés en generar un conjunto de reformas en el sistema educativo, a tal punto que se comunicaba la intención de realizar una "revolución educativa", pero el periodo analizado no presenta cambios relevantes en las políticas educativas. Se anunciaron en diferentes momentos un conjunto de planes educativos como "el Plan Maestro" y "Secundaria 2030" que no lograron registrar avances considerables. El área de Evaluación fue la única que impulsó cambios como el reemplazo del Operativo Nacional de Evaluación (ONE) por el Operativo Aprender. A su vez, un elemento de interés al financiamiento educativo en este periodo es que en el año 2017 el Ministerio de Educación nacional resolvió no llamar a la paritaria nacional docente, incumpliendo la Ley de Educación Nacional.

Durante los cuatro años que atravesaron a esta gestión no se han realizado cambios sustanciales en el marco normativo que organiza el financiamiento educativo. No obstante, sí se registran algunas cuestiones novedosas en la dinámica del financiamiento del sector.

Cuadro 5. Inversión consolidada en educación por nivel de gobierno como porcentaje del PBI (2016-2019)

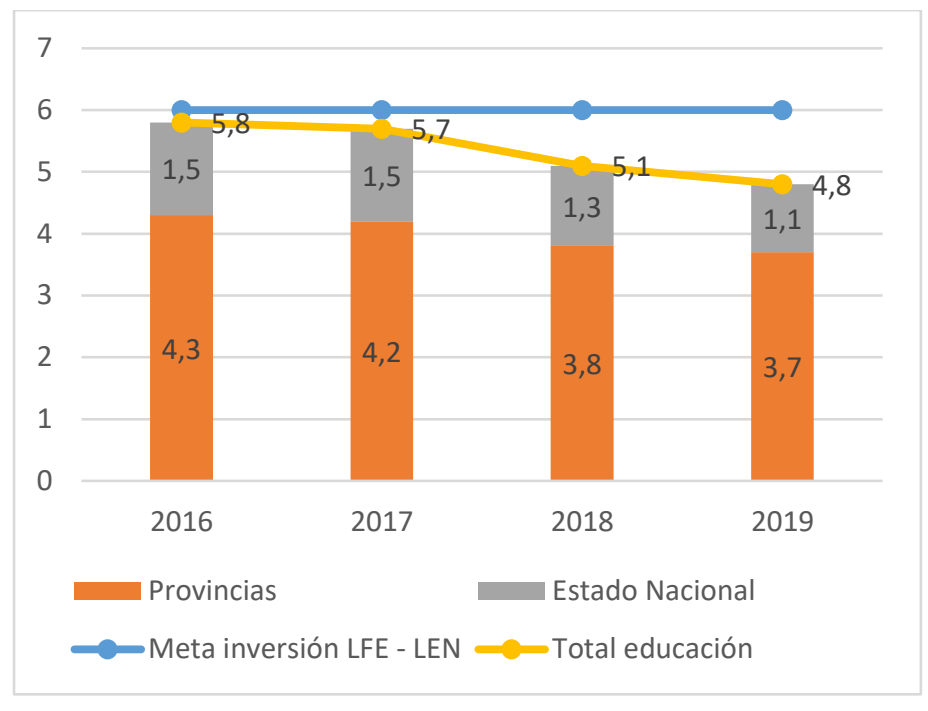

Fuente: Elaboración propia en base a los datos de la Secretaría de Política Económica, del Ministerio de Hacienda para los años 2016 - 2017 y a datos de Clauss y Bucciarelli (2020) para los años 2018 - 2019.

Si seguimos los datos que muestra el cuadro 5 podemos ver que la inversión consolidada en educación por nivel de gobierno muestra cambios. En principio, la dinámica de 2016 a 2019 nos permite ver que, a lo largo del periodo la inversión consolidada cae, pero esta caída no es homogénea. En los primeros dos años la inversión consolidada registra una caída en la inversión que realizan las provincias de $4,3 \%$ en 2016 a 4,2\% en 2017, manteniéndose constante la participación a nivel nacional. En cambio, en los últimos dos años la caída se acelera tanto a nivel de la participación nacional como de las provincias, aunque en distintas proporciones. Por su parte, Nación registra una caída de 0,2\% en 2018 y $0,2 \%$ del PBI más en 2019. En cambio, las provincias registran en conjunto una caída mayor que va de $4,2 \%$ a $3,8 \%$ puntos del PBI en 2018 y desciende a 3,7\% en 2019 lo que muestra efectivamente que a las provincias le fue mucho más difícil mantener su nivel de inversión. Si miramos estos datos juntos a los del apartado anterior, podemos ver que la relación entre la inversión y las metas de financiamiento planteadas, en un principio, en la LFE y, posteriormente, en la LEN solo fueron alcanzadas, como dijimos, en 2009 y 2015 , comenzando ampliarse la brecha entre las metas y la inversión a partir de 2016, pero sobre todo y de modo sensible a partir de 2018 continuando en el 2019.

De principio a fin del periodo la inversión educativa registra un desplome de aproximadamente un $17 \%$ en términos reales (5,8\% a $4,8 \%$ del $\mathrm{PBI})$. No podemos dejar de notar que el momento en el que el financiamiento del sector comienza caer sensiblemente es el momento en el que el elevado nivel de endeudamiento público comienza a presionar fuertemente los recursos con los que cuenta el Estado Nacional para afrontar sus compromisos y, a su vez, momento en el que la economía nacional comienza un periodo recesivo presionada, justamente, por el nivel de endeudamiento y las tensiones cambiarias que se comienzan a expresar como devaluaciones recurrentes de la moneda. Un elemento que permite entender las razones del desplome de la inversión educativa, al menos - y principalmente - a la parte que concierne al Estado Nacional está en la ratio entre la función de educación y la de deuda pública en las cuentas del Estado nacional. En estos es posible observar que hay un crecimiento exponencial de la deuda en todo este periodo de gobierno que, según UNIPE (2018), pasan de tener una relación de 0,9 pesos a servicios de deuda por cada peso destinado a educación a 3,3 pesos a servicios de deuda por cada peso destinado a educación. 
REVISTA DE LA ESCUELA DE CIENCIAS DE LA EDUCACIÓN, AÑo 17, NRO. 16, VOL. 1, ENERO A JUNIO dE 2021. PÁGINAS 77-88. ISSN 2362-3349 (EN LíNEA). EL FINANCIAMIENTO EDUCATIVO ARGENTINO EN LOS ÚLTIMOS TREINTA AÑOS (1989 - 2019). RUPTURAS Y CONTINUIDADES EN LAS TRES ÚLTIMAS DECADAS. PEDRO, FIORUCCI. ALEJANDRO, ANTON.

\section{Conclusiones}

En los 30 años analizados del financiamiento del sistema educativo argentino hemos registrado tres períodos políticos y económicos que han sido el contexto macro en el que, cada uno a su modo, le ha impreso una dinámica particular al financiamiento del sector. Todos, a su vez, han sido periodos políticos y económicos que han asumido diferentes políticas para orientar el sistema educativo.

Los rasgos del periodo neoliberal se han caracterizado por mantener una inversión educativa en crecimiento que muestra un alza muy significativa pasando de un $3 \%$ a un $5 \%$ del PBI. Este incremento tiene el antecedente de registrarse sobre el suelo que tocó el financiamiento del sector en el contexto de crisis fiscal y financiera que afrontó el país a fines de los '80. A su vez, el crecimiento de casi 2 puntos porcentuales se dio en el proceso de transferencia de servicios educativos secundarios y terciarios no universitarios del Estado Nacional a las provincias, lo que implicó en los hechos una reducción sensible del nivel de inversión que realizaba nación y su consecuente traslado hacia las provincias. Esta transferencia implicó la consolidación de un proceso de descentralización del sistema educativo que perdura hasta la actualidad con un esquema federal de financiamiento al sector.

El colapso del modelo de la convertibilidad se da de la mano de una caída de 1,5 puntos del PBI en el sector educativo. Este proceso comienza a revertirse posteriormente en el periodo neo-desarrollista, principalmente a través de la sanción en el año 2005 de la LFE 26.075 y posterior a 2010 bajo la normativa de la Ley de Educación Nacional. Este proceso fue acompañado de un ciclo económico que tuvo como rasgos principales la reactivación de la economía y un crecimiento económico sostenido, que posibilitaron tanto recomponer los recursos públicos a disposición del Estado como incrementar su intervención y participación en distintas áreas de la sociedad. Si bien en este período se inició un proceso de planificación de la estrategia para mejorar el financiamiento del sector por unos pocos años, se continuó con el sistema descentralizado del período neoliberal y no se logró revertir los desequilibrios fiscales entre las jurisdicciones y el estado nacional.

El tercer periodo inicia a fines de 2015 y concluye a fines de 2019, registrando una notable reversión de los avances alcanzados en la última década en términos de financiamiento del sector. Esto puede observarse en la caída de 5,8\% a 4,8\% del PBI para educación en cuatro años, descendiendo la participación tanto del estado nacional como de las provincias. En este proceso no sólo hay que tener en cuenta el ajuste que atraviesa el sector sino la disputa mayor que comienza a registrarse entre los recursos públicos con que cuenta el Estado y las exigencias que le plantea al gasto público el nivel de endeudamiento alcanzado. Además de este proceso, no se volvió a planificar una estrategia de inversión con responsabilidades claras para cada nivel de gobierno ni se revirtió la tendencia de desequilibrios fiscales entre niveles de gobierno, ambos aspectos que ya venían desarrollandose en el periodo anterior.

En cuanto a la participación del Estado Nacional vemos que si bien aumentó un 25\% la inversión en relación al $\mathrm{PBI}$ (de 1,2\% a un 1,5\%) lo cual puede interpretarse como un interés de recuperar centralidad en el financiamiento del sistema educativo, no se logró alcanzar la meta de financiar en un 40\% a educación. A su vez, este proceso de crecimiento de la inversión comienza a revertirse en el último periodo estudiado, que lleva esta participación del Estado Nacional de 1,5\% del PBI en 2016 a un 1,1\% en 2019. Esta dinámica nos coloca ante un proceso en el cual avizoramos profundos desafíos para cambiar la tendencia de los últimos años y mejorar el financiamiento educativo con mayor participación del Estado Nacional.

\section{Referencias bibliográficas}

Aronskind, R. (2015). Intuiciones y confrontaciones. Para pensar la política económica kirchnerista. En: Revista Márgenes. Revista de economía política. Los Polvorines: UNGS.

Basualdo, E. (2011). Sistema político y modelo de acumulación. Tres ensayos sobre la Argentina actual. Buenos Aires: Cara o Ceca.

Basualdo, E. (2013). El legado dictatorial. El nuevo patrón de acumulación de capital, la desindustrialización y el ocaso de los trabajadores. En: Verbisky, H. y Bohoslavsky, J. P. Cuentas pendientes: Los cómplices económicos de la dictadura. Buenos Aires: Siglo XXI.

Basualdo, E. y Manzanelli, P. (2016). Régimen de acumulación durante el ciclo de gobiernos kirchneristas. En: Revista Relidad Económica № 304 pp, 6-40, IADE, Buenos Aires.

Bezem, P.; Mezzadra, F. y Rivas, A. (2012). Monitoreo de la Ley de Financiamiento Educativo. Buenos Aires: CIPPEC.

Bona, L. (2016). Del kirchnerismo al macrismo: ¿nueva hegemonía y bloque en el poder? IX Jornadas de Sociología de la UNLP, Ensenada, Argentina.

CIFRA (2016). La naturaleza política y económica de la alianza Cambiemos. DT № 15. Buenos Aires, Argentina.

Claus, A. y Bucciarelli, M. (2020). El financiamiento educativo en Argentina: el desafío de la equidad bajo un esquema federal. Documento de Trabajo No194. Buenos Aires: CIPPEC

Feldfeber, M. (2000). Una transformación sin consenso: apuntes sobre la política educativa del gobierno de Menem". En: Versiones, № 11. Universidad de Buenos Aires: Ediciones Novedades Educativas.

Forcinito, K. y Tolon, G. (2009). Reestructuración neoliberal y después. 1983-2008: 25 años de economía argentina. Los Polvorines: UNGS.

Morduchowicz, A. (2002). El financiamiento educativo en Argentina: problemas estructurales, soluciones coyunturales. UNESCO

Morduchowicz, A. (2019). El financiamiento educativo argentino. En: Propuesta Educativa, Año 28, № 52, noviembre, pp. 11-23.

Nahon, C. (2010). Transformaciones económicas en la década del noventa en la Argentina: la consolidación de la valorización financiera. En: Arceo, N. y Socolovsky. Y. Desarrollo económico, clase trabajadora y luchas sociales en la Argentina contemporánea. Buenos Aires: IEC-CONADU

Novaro, M. (2010). Historia de la Argentina 1955-2010. Buenos Aires: Siglo XXI. 
REVISTA DE LA ESCUELA DE CIENCIAS DE LA EdUCACIÓN, AÑo 17, NRO. 16, VOL. 1, ENERO A JUNIO DE 2021. PÁGINAS 77-88. ISSN 2362-3349 (EN LínEA). EL FINANCIAMIENTO EDUCATIVO ARGENTINO EN LOS ÚLTIMOS TREINTA AÑOS (1989 - 2019). RUPTURAS Y CONTINUIDADES EN LAS TRES ÚLTIMAS DECADAS. PEDRO, FIORUCCI. ALEJANDRO, ANTON.

Rivas, A.; Vera, A. y Bezem, P. (2010). Radiografía de la educación argentina. Buenos Aires: CIPPEC.

Rivas, A y Dborkin, D (2018). ¿Qué cambió en el financiamiento educativo en Argentina? Documento de trabajo №162. Febrero 2018. CIPPEC: Argentina

Saforcada, F. (2012). Las políticas de autonomía escolar en la década del 90: el caso Nueva Escuela Argentina para el siglo XXI (Tesis de Maestría). FLACSO.

Suasnábar (1999). Resistencia, cambio y adaptación en las universidades nacionales. En: Tiramonti, G.; Suasnábar, C. y Seoane, V. Políticas de modernización universitaria y cambio institucional. La Plata: FAHCE - UNLP.

Torrado, S. (1992). Estructura social de la Argentina: 1945-1983. Ediciones de la Flor: Buenos Aires

UNIPE; FFYL-UBA; CCC (2018). El presupuesto educativo entre 2016 y 2019. Degradación del financiamiento y debilitamiento de las políticas nacionales. Octubre 2018. Argentina.

Varesi, G. (2011). Argentina 2002-2011: neodesarrollismo y radicalización progresista. En: Revista Realidad Económica, № 264. IADE: Buenos Aires.

Varesi, G. (2016). La contraofensiva neoliberal: estrategia político-económica de reestructuración societaria en el primer semestre de Macri. IX Jornadas de Sociología de la UNLP, Ensenada, Argentina.

Varesi, G. (2018). De la crisis del régimen neo-desarrollista y la hegemonía kirchnerista al triunfo de Cambiemos. En: Revista Temas y debates. 35, año 22, enero-junio 2018, pp.13-38.

Vera, A. y Bilbao, R. (2010). La apuesta de invertir para una mejor educación: evaluando la ley de financiamiento educativo en la Argentina 2006-2010. Buenos Aires: CIPPEC.

Vilas, C. (2007). ¿Hacia atrás o hacia adelante? La revalorización del Estado después del "consenso de Washington. En: Perspectivas, Sao Paulo, Vol, 32. jul./dez. 